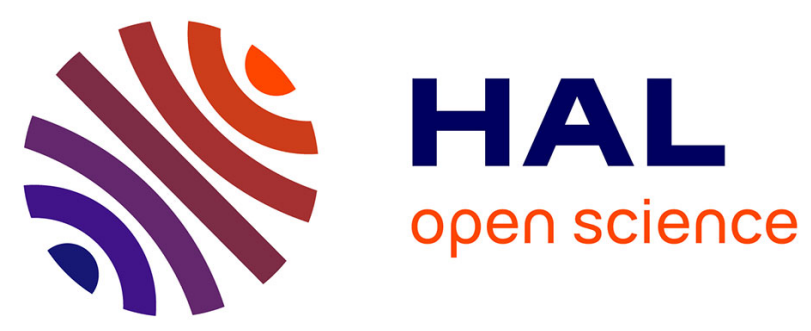

\title{
Left amygdala volume and brain tissue pulsatility are associated with neuroticism: an MRI and ultrasound study
}

Marta Andrea Siragusa, Thomas Rufin, Robert Courtois, Catherine Belzung, Frédéric Andersson, Bruno Brizard, Paul-Armand Dujardin, Jean-Philippe Cottier, Frédéric Patat, Jean-Pierre Remenieras, et al.

\section{To cite this version:}

Marta Andrea Siragusa, Thomas Rufin, Robert Courtois, Catherine Belzung, Frédéric Andersson, et al.. Left amygdala volume and brain tissue pulsatility are associated with neuroticism: an MRI and ultrasound study. Brain imaging and behavior (Brain Imaging Behav), 2020, pp.1499-1507. 10.1007/s11682-020-00348-w . hal-03265218

\section{HAL Id: hal-03265218 \\ https://hal.science/hal-03265218}

Submitted on 5 Feb 2022

HAL is a multi-disciplinary open access archive for the deposit and dissemination of scientific research documents, whether they are published or not. The documents may come from teaching and research institutions in France or abroad, or from public or private research centers.
L'archive ouverte pluridisciplinaire HAL, est destinée au dépôt et à la diffusion de documents scientifiques de niveau recherche, publiés ou non, émanant des établissements d'enseignement et de recherche français ou étrangers, des laboratoires publics ou privés. 


\title{
Left amygdala volume and brain tissue pulsatility are associated with neuroticism: an MRI and ultrasound study
}

\author{
Marta Andrea Siragusa ${ }^{1}$ (D) Thomas Rufin ${ }^{2} \cdot$ Robert Courtois $^{3,4} \cdot$ Catherine Belzung $^{1} \cdot$ Frédéric Andersson $^{1}$. \\ Bruno Brizard ${ }^{1}$ - Paul-Armand Dujardin ${ }^{5}$. Jean-Philippe Cottier ${ }^{1,2}$. Frédéric Patat ${ }^{1,2,5}$ • Jean-Pierre Réméniéras ${ }^{1}$. \\ Valérie Gissot $^{5} \cdot$ Wissam El-Hage ${ }^{1,2,5} \cdot$ Vincent Camus $^{1,2} \cdot$ Thomas Desmidt $^{1,2}$
}

Published online: 6 August 2020

(C) Springer Science+Business Media, LLC, part of Springer Nature 2020

\begin{abstract}
Brain changes associated with the personality trait of neuroticism have been partly elucidated. While subcortical brain volume changes, especially a larger amygdala, appear consistent in high neuroticism, functional changes, such as cerebral blood flow (CBF) differences, have shown conflicting results, possibly because of the limitations in methods of CBF measurement. In our study, we investigated changes in amygdala volume and CBF-related function associated with neuroticism in healthy and depressed subjects using both conventional magnetic resonance imaging (MRI) measures of brain volume and the innovative technique of ultrasound Tissue Pulsatility Imaging (TPI), which has a high level of detection in measuring brain tissue pulsatility (BTP). Middle-aged females with depression $(n=25)$ and without depression $(n=25)$ underwent clinical examination, magnetic resonance imaging (MRI) and ultrasound assessment (TPI). Neuroticism was positively associated with left amygdala volume and mean BTP in individuals without depression, in both simple and multiple regressions that included potential confounding factors such as age and body mass index. No association was found in the depressed group. We confirmed the role of the left amygdala in the brain physiology of neuroticism in nondepressed individuals. Moreover, we identified a novel mechanism associated with high neuroticism, namely BTP, that may reflect greater CBF and account for the increased risk of cerebrovascular disease in individuals with high neuroticism. Because neuroticism is considered a risk factor for depression, our paper provides potential objective biomarkers for the identification of subjects at risk for depression.
\end{abstract}

Keywords Brain tissue pulsatility $\cdot$ Tissue pulsatility imaging $\cdot$ Amygdala volume $\cdot$ Neuroticism

\section{Introduction}

Neuroticism is a personality trait characterized by a general trend toward negative affectivity (Clark and Watson 2008; Costa and McCrae 1992). It has been found to constitute a marker of vulnerability for major depression (Ormel et al.

Thomas Desmidt

t.desmidt@chu-tours.fr

UMR 1253, iBrain, Inserm, Université de Tours, Tours, France

CHRU de Tours, Tours, France

3 CRIAVS Centre-Val de Loire, CHRU de Tours, Tours, France

4 Département de Psychologie, EE 1901 'Qualipsy' (Qualité de vie et Santé psychologique), Université François Rabelais de Tours, Tours, France

5 CIC 1415, CHU de Tours, Inserm, Tours, France
2013). Several epidemiological studies have reported a significant association between neuroticism and major depressive disorder (MDD), including a recent meta-analysis that found a large effect size (Cohen's $d=1.54$ ) in the association between neuroticism and MDD (Kotov et al. 2010). Based on such evidence, the DSM-5 now considers high levels of neuroticism as a risk factor for the development of MDD (DSM 2013).

Some neuroimaging studies have shown that changes in brain structure and function with neuroticism can act as a risk factor for MDD. Notably, a meta-analysis of brain structural changes with neuroticism found a trend for a larger left amygdala with high neuroticism (Mincic 2015), although a number of studies found no specific association (Gray et al. 2018) or associations involving the right amygdala (Holmes et al. 2012), sometimes as a negative correlation (Omura et al. 2005). A reduction in the volume of the hippocampus has also been reported (Yamasue et al. 2008; Kempton et al. 2011) in 
individuals with high neuroticism. Studies using functional magnetic resonance imaging (fMRI) have reported that individuals with higher neuroticism show greater activation in limbic structures such as the amygdala in response to arousing stimuli (Eysenck 1967; Eysenck 1990; Wei et al. 2014). Positron emission tomography (PET) studies have also identified significant associations between cerebral blood flow $(\mathrm{CBF})$ and neuroticism in limbic regions, such as an increased activity of the right hippocampus associated with neuroticism (Sutin et al. 2010).

Interestingly, the changes associated with neuroticism seem to share some brain structural and functional characteristics with those associated with MDD, which may account for the role of neuroticism as a risk factor for MDD. Notably, similar subcortical volume changes have been found in neuroticism and MDD that specifically involve the amygdala (Hamilton et al. 2008) and hippocampus (Schmaal et al. 2015). Functional changes may also be involved in the association between neuroticism and MDD, as elevated baseline activity in the amygdala and heightened amygdala responsivity to affective stimuli have been found in both high neuroticism and MDD (Hamilton et al. 2008; Drevets et al. 1992). Changes in CBF may also be involved in the brain mechanisms of neuroticism as a risk factor for MDD. However, studies investigating CBF as assessed by PET and ASL-MRI have reported contrasting results, with both increased and decreased CBF perfusion found in various brain regions such as the limbic areas (Liao et al. 2017; Ho et al. 2013; Sutin et al. 2010; Lui et al. 2009). Several reasons may account for the inconsistent results concerning CBF in MDD and neuroticism, including limitations in the neuroimaging techniques classically used to assess CBF (PET and ASL-MRI), such as a relatively low spatiotemporal resolution, which can lead to misidentification of subtle CBF changes.

Recent advances in ultrasound (US) imaging technology have allowed the development of innovative neuroimaging techniques to accurately detect biomechanical brain properties related to $\mathrm{CBF}$, such as the so-called Tissue Pulsatility Imaging (TPI) that our group has recently used to identify subtle changes in the Brain Tissue Pulsatility (BTP) of individuals with MDD (Desmidt et al. 2017). TPI is similar to transcranial Doppler (TCD), except that rather than focusing on only large arteries, it relies on the Echo-B mode of a modern US scanner to measure the pulsatile movements of large brain regions, which allows a very high spatiotemporal level of detection (micrometers/millisecond) of brain volume changes related to pulsatile CBF. Periodic changes in blood volume cause the brain to expand and relax over the cardiac cycle, and TPI measures the pulsatile signals from thousands of sample volumes in an US image plane, in accordance with the principle of plethysmography (Kucewicz et al. 2004). TPI has been validated on phantoms (Kucewicz et al. 2004) and on healthy volunteers. It has been shown that BTP is increased during visual stimulation within the posterior region of the brain (Kucewicz et al. 2007) and decreased in the posterior temporal lobe during hyperventilation (Kucewicz et al. 2008), which suggests that BTP is closely related to CBF changes and cerebrovascular reactivity, in accordance with the notion that pulsatile $\mathrm{CBF}$ and cerebrovascular functioning are major determinants of brain pulsatility (Wagshul et al. 2011). More recently, our team also found that TPI can indicate brain functional changes in some pathological conditions, including cerebrovascular lesions, orthostatic hypotension and depression. Specifically, we found that midlife depressed females showed greater global BTP compared to healthy controls and individuals remitted from depression (Desmidt et al. 2017).

The goal of our study was to investigate associations among neuroticism, amygdala volume - the most consistently observed subcortical volume change associated with neuroticism - and BTP in a group of healthy and depressed individuals. Because BTP was shown to be greater in MDD and because amygdala volumes have been found to be changed in MDD, we hypothesized that a higher level of neuroticism, which is a strong risk factor for MDD, would be associated with greater BTP and a larger amygdala in both depressed and healthy subjects.

\section{Methods}

\section{Participants}

Fifty females aged 18 to 55 years were recruited. Participants were depressed subjects and healthy controls from the EMPHILINE project (NCT02026622 on clinicaltrials.gov), whose principal objective was to investigate the cardiovascular and cerebrovascular reactivity in depression during emotional tasks; the baseline data are reported in the present article. To reduce variability, only females were recruited because previous findings have found significant differences in cerebrovascular properties between agematched males and females. Subjects in the depressed group (group D; $n=25$ ) were inpatients or outpatients from the psychiatric ward of the University Hospital of Tours, France. Psychiatrists (TD, WEH) diagnosed the patients as depressed according to the DSM-IV criteria for MDD, with a MADRS score higher than 21. Subjects in the control group (group C; $n=25$ ) were recruited from the local community and from the records of the Clinical Investigation Center of the Hospital of Tours, France. They had no history of MDD or any psychiatric disorder and had a current MADRS score lower than 9 . The two groups were matched for age. Noninclusion criteria for all subjects were: 1) history of psychotic, bipolar, or substance-abuse disorders or suspicion of severe cognitive impairment (MMSE <25), 2) history of severe cardiovascular 
diseases (myocardial infarction, arrhythmia, etc.) or neurological disorders (stroke, brain tumor, severe concussion, migraine, etc.), 3) current unstable medical condition, 4) current use of beta-blockers or antipsychotics, 5) smoking over 10 packs/year, 6) auditory or visual impairments, 7) pregnancy or unreliable contraception, 8) contraindication for MRI and 9) legal guardianship. Informed consent was obtained from all subjects, and the study protocol was approved by the local human ethical committee.

\section{Clinical assessments}

Either a psychiatrist (TD) or a trained medical doctor (VG) performed the clinical assessments. Medical history and medication intake were recorded. The clinical assessment included blood pressure (measured immediately before the US assessment), height and weight measurements. Psychometric assessments included 1) the Structured Clinical Interview for DSMIV (SCID) for current or lifetime MDD; 2) the MADRS for severity of depression; 3) the Mini-Mental State Examination (MMSE) to assess global cognitive functioning and screening for dementia; and 4) the Revised NEO Personality Inventory (NEO PI-R), a 240-item questionnaire used to measure personality traits based on the Big Five Theory (Costa and McCrae 1992). Answers are presented on a five-point Likert scale; in the present study, only the neuroticism score was reported.

\section{Magnetic resonance imaging protocol}

MRI scanning sessions were performed immediately after the psychometric assessment on a 3 Tesla Siemens Verio scanner (Siemens AG, Erlangen, Germany). A high-resolution T1weighted MRI 3D volume sequence (192 contiguous sagittal slices; $1 \mathrm{~mm}$ slice thickness; TR $=1.9 \mathrm{~s} ; \mathrm{TE}=2.42 \mathrm{~ms}$; TI = $0.9 \mathrm{~s} ; \mathrm{FA}=9^{\circ}$; in-plane resolution: $1 \times 1 \mathrm{~mm}$ ) was acquired for each subject. Additional sequences (T2-weighted and fluid-attenuated inversion recuperation) were acquired and analyzed to rule out concomitant diseases such as ischemic stroke and susceptibility artifacts from prior hemorrhages or space-occupying lesions. Severe white matter lesion load (Fazekas grade 3) was used as an exclusion criterion.

Structural image processing Cortical reconstruction and volumetric segmentation were performed with the Freesurfer image analysis suite, which is documented and freely available for download online (http://surfer.nmr.mgh.harvard.edu/). The technical details of these procedures are described in prior publications (Reuter et al. 2010; Fischl et al. 2004a, b; Ségonne et al. 2004; Fischl et al. 2002). Briefly, this processing includes removal of nonbrain tissue using a hybrid watershed/surface deformation procedure (Ségonne et al. 2004), automated Talairach transformation, segmentation of the subcortical white matter and deep gray matter volumetric structures (including the hippocampus, amygdala, caudate nucleus, putamen, and ventricles) (Fischl et al. 2004a, 2002). In the present study, only amygdala volumes were analyzed. Freesurfer provided the estimated Total Intracranial Volume (eTIV), which was labeled as ICV in our study. To limit interindividual variability in amygdala volumes due to differences in ICVs, the absolute volume of the amygdala was divided by ICV for group comparisons and testing for associations. Controlling the absolute volume of the amygdala with ICV as a covariate in multiple regression analyses was also considered in our study as another method for associations testing.

\section{Ultrasound protocol}

The US protocol was performed immediately following the MRI sessions on an Antares medical scanner (Siemens Healthcare, Germany) by a single biophysics technician (B.B.) trained for TCD, who was blinded to the MRI results. Transcranial acquisitions were performed with a PX4-1 phased-array transducer (Siemens Healthcare; $1.82 \mathrm{MHz}$ emission frequency, $70^{\circ}$ field of view, $112 \times 3$ elements $(1.5$ D)). Measurements were performed through the right temporal bone window, which is the thinnest bone of the skull, with the probe positioned perpendicularly to the skull and maintained by a mechanical holder to reduce artifacts caused by the operator's and the subject's movements. Subjects were asked to remain in the supine position, to try not to move, to close their eyes and to breathe normally.

The US scanner was first switched on in Doppler mode for TCD acquisition. Color Doppler was used to center the US beam on the right MCA. The pulsatility index (PI - ratio of the difference between maximal and minimal velocities over mean velocity) was acquired and automatically computed by the scanner from a $10 \mathrm{~s}$ Doppler sequence.

The US scanner was then switched to Echo-B mode to perform TPI measurements centered on the MCA. To limit US attenuation, we adjusted the depth of the acquisition between 3 and $9 \mathrm{~cm}$. With this configuration, we explored the circle of Willis and a transverse slice of the temporal hemispheres.

For each subject, the protocol consisted of 4 acquisitions of $10 \mathrm{~s}$ repeated every $26 \mathrm{~s}$ with an acquisition frame rate of 30 images/s (total of 297 frames). The US scanner provided direct access to beam-formed radiofrequency (RF) lines, which were used to estimate BTP. The data were then downloaded for offline analysis with MATLAB software (MathWorks Inc., USA).

Tissue Pulsatility imaging signal processing The signal processing used to extract Brain Tissue Pulsatility measurements from Echo-B signals has been extensively described 
previously (Desmidt et al. 2017). Briefly, axial BTP was estimated using a 1D-intercorrelation method between successive acquisition frames. The maximum of the normalized intercorrelation coefficient, Txy/sqrt(TxxTyy), was used to estimate the delay between successive kernels on RF lines at a specific depth. The normalized correlation coefficient had to be higher than 0.7 to validate the measurement. A kernel size of 8 periods (176 points, 4 wavelengths, $3.3 \mathrm{~mm}$ at $1.82 \mathrm{MHz}$ ) was chosen. Parabolic interpolation was performed around the 3 maximum points of the 1D correlation to improve the precision of the displacement measurement. With the signal-tonoise Ratio obtained after propagation through the skull, the noise level of BTP was $0.8 \mu \mathrm{m}$. For better spatial discretization along RF lines, an overlap rate of $80 \%$ of the kernel was computed, resulting in a $660 \mu \mathrm{m}$ spatial discretization. A bandpass filter (low cutoff $=0.75 \mathrm{~Hz}$; high cutoff $=5.0 \mathrm{~Hz}$ ) was then applied to focus on displacements related to heart rate and to filter out slow and fast movements due to respiration and artifacts, respectively.

We obtained a 3D matrix of BTP: axial displacements along the $\mathrm{z}$-axis (RF lines), $\mathrm{x}$-axis (the 112 elements of the probe) and time. The temporal evolution of BTP was analyzed at each position of the 2D plane (curves of the displacement as a function of time). We used two criteria to filter out artifacts and focus on physiological signals, which are supposed to be stable in terms of periodicity and amplitude. The first criterion was used to investigate the periodicity of the brain pulsations and consisted of the ratio of the second maximum peak over the central peak (SMP/CP) of the temporal autocorrelation function of each kernel. If the SMP/CP ratio was greater than 0.6 , the record was validated and compared to the second criterion. The threshold of 0.6 was chosen based on previous studies (Desmidt et al. 2017; Ternifi et al. 2014; Desmidt et al. 2011). The second criterion was used to investigate the difference in amplitudes between pulsations in each curve. The cumulated standard deviation (CSTD) of the pulsations was calculated to determine the data dispersion and was normalized to the peak-to-peak amplitude (Umean) of the mean temporal curve. If the ratio CSTD/Umean was lower than 0.25 , the record was validated and rejected otherwise. The threshold of 0.25 was chosen for the same reasons as the first criterion.

From this final matrix, we calculated the root mean square of 2 curves, MaxBTP and MeanBTP, corresponding, respectively, to the curve with the maximum mean peak-to-peak amplitude (averaged between cycles) and to the average of all curves in the matrix. MaxBTP and MeanBTP thus correspond to the pulsatility of, respectively, the largest and the mean pulsatility in the whole region of acquisition.

\section{Statistical analyses}

Shapiro-Wilk tests determined that amygdala volumes, ultrasound and neuroticism measures were normally distributed.
We therefore performed parametric analysis for group comparisons (Student's t tests).

We applied simple linear regression analyses in both groups to identify 1) associations of amygdala volumes with neuroticism and 2) associations of brain pulsatility and TCDPI with neuroticism. The dependent variables were the measurements of brain tissue pulsatility and TCD-PI and the amygdala volumes, and the independent variable was the neuroticism score. We also applied multiple linear regressions in both groups including age, BMI and antidepressant intake as potential confounding factors. Notably, associations between amygdala volume and neuroticism were investigated using both 1) the ratio of the amygdala volume over ICV and 2) the amygdala volume corrected with ICV as a covariate in a multiple linear regression, to test for potential discrepancies with these two methods of controlling for interindividual variability in amygdala volumes due to differences in ICVs.

\section{Results}

\section{Comparisons between groups}

Demographic and clinical characteristics are shown in Table 1. There was no difference in age or cardiovascular risk factors between the groups. As expected, antidepressant intake, MADRS score and neuroticism score were significantly greater in group D compared to group $\mathrm{C}\left(p<10^{-4}\right)$.

MRI and ultrasound results are shown in Table 2. R and L AMY were significantly lower in group D compared to group C. ICV was greater in group D compared to group C, even if the difference was at the threshold of statistical significance. The mean BTP was significantly greater in group D compared to group C. There was no significant difference in TCD-PI between the two groups.

\section{Linear regressions}

Simple linear regressions between neuroticism and 1) AMY and 2) BTP measures in group $C$ and D are shown in Fig. 1. Neuroticism was significantly associated with meanBTP in group $\mathrm{C}$ [beta $=0.134, p=0.02$ ]. Neuroticism was also associated with $\mathrm{L}$ AMY in group $\mathrm{C}$, both using the absolute amygdala volume [beta $=9.033, p=0.018]$ and the ratio of the amygdala volume over ICV (beta $=9.105 \mathrm{e}^{-6}, p<10^{-4}$ ). We found no significant association between neuroticism and TCD-PI in any of the two groups [beta $=0.001, p=0.917$ in group $\mathrm{C}$; beta $=-0.006, p=0.096$ in group $\mathrm{D}]$.

In multiple regressions neither age, BMI nor antidepressant intake modified the significance of the associations between neuroticism and 1) AMY or 2) BTP measures. When including ICV as an independent variable with the absolute amygdala volume as the dependent variable, the association of 
Table 1 Demographic and clinical characteristics of the population

\begin{tabular}{|c|c|c|c|}
\hline & $\begin{array}{l}\text { Depressed patients } \\
(\mathrm{n}=25)\end{array}$ & $\begin{array}{l}\text { Controls } \\
(\mathrm{n}=25)\end{array}$ & $\mathrm{p}$ value \\
\hline Age & $39.1(11.4)$ & $39.7(11.3)$ & 0.862 \\
\hline \multicolumn{4}{|l|}{ Cardiovascular risk factors } \\
\hline Smoking & $11(44 \%)$ & $5(20 \%)$ & 0.069 \\
\hline BMI $\left(\mathrm{kg} / \mathrm{m}^{2}\right)$ & $23.8(4.9)$ & $23.0(3.5)$ & 0.520 \\
\hline High blood pressure & $2(8 \%)$ & $2(8 \%)$ & 1 \\
\hline High cholesterol & $2(8 \%)$ & $1(4 \%)$ & 0.552 \\
\hline Diabetes & 0 & 0 & - \\
\hline Baseline SBP (mmHg) & $122.3(14.4)$ & $119.8(13.7)$ & 0.529 \\
\hline Baseline DBP (mmHg) & $76.3(11.9)$ & $72.7(10.3)$ & 0.259 \\
\hline \multicolumn{4}{|l|}{ Medication intake } \\
\hline Antidepressant & $11(44 \%)$ & 0 & 0.0004 \\
\hline Anxiolytic & $7(28 \%)$ & $1(4 \%)$ & 0.053 \\
\hline Mood stabilizer & $3(12 \%)$ & 0 & 0.193 \\
\hline Antihypertensive & $1(4 \%)$ & $1(4 \%)$ & 1 \\
\hline \multicolumn{4}{|l|}{ Depression characteristics } \\
\hline Age at onset & $27.9(10.5)$ & - & - \\
\hline Number of episodes & $2.0(1.6)$ & - & - \\
\hline Illness duration (weeks) & $32(36.5)$ & - & - \\
\hline MADRS & $31.5(5.2)$ & $1.2(1.6)$ & $<10^{-4}$ \\
\hline \multicolumn{4}{|l|}{ NeoPI-R } \\
\hline Neuroticism & $61.8(11.3)$ & $47.1(9.9)$ & $<10^{-4}$ \\
\hline
\end{tabular}

Values are expressed as the mean (standard deviation) or $\mathrm{n}(\%)$. Comparisons were performed with $t$ tests (quantitative data) or chisquared tests (qualitative data)

$B M I$, Body mass index; $D B P$, Diastolic blood pressure; MADRS, Montgomery and Asberg Depression Rating Scale; NeoPI, Neopersonality Inventory; $S B P$, systolic blood pressure.

Table 2 Comparisons of MRI and TPI measures between the two groups

\begin{tabular}{llll}
\hline & $\begin{array}{l}\text { Depressed patients } \\
(\mathrm{n}=25)\end{array}$ & $\begin{array}{l}\text { Controls } \\
(\mathrm{n}=25)\end{array}$ & p value \\
\hline MRI & & & \\
ICV $\left(\mathrm{cm}^{3}\right)$ & $13,551(1013)$ & $12,986(964)$ & 0.049 \\
L AMY $(\%)$ & $0.109(0.008)$ & $0.119(0.013)$ & 0.003 \\
R AMY $(\%)$ & $0.113(0.01)$ & $0.123(0.012)$ & 0.003 \\
Ultrasound & & & \\
Mean BTP $(\mu \mathrm{m})$ & $9.7(4.1)$ & $7.3(2.9)$ & 0.021 \\
TCD-PI & $0.92(0.20)$ & $0.96(0.26)$ & 0.581 \\
\hline
\end{tabular}

Values are expressed as the mean (standard deviation). Amygdala values are expressed as relative volumes (volume/ICV). Comparisons were performed with $\mathrm{t}$ tests

$I C V$, Intracranial volume; $A M Y$, Amygdala; $B T P$, Brain tissue pulsatility; $M R I$, Magnetic resonance imaging; $T C D-P I$, transcranial Dopplerpulsatility index neuroticism with L AMY became stronger [beta $=12.198$, $p<10^{-4}$ ] and ICV correlated with L AMY [beta $=0.001$, $p=0.001]$. Finally, we found no significant association among neuroticism, AMY and BTP in group D (Fig. 2).

\section{Discussion}

Our principal finding was that neuroticism was positively correlated with both left amygdala volume and brain pulsatility (BTP) in our sample of middle-aged healthy females, independent of age, body mass index or antidepressant use. No significant association was found between neuroticism and both amygdala volume and pulsatility measures in depressed individuals.

Our result of a highly significant association between left amygdala and neuroticism may appear surprising, especially because previous studies have inconsistently reported such an association. Indeed, several previous studies found no association between amygdala volume and neuroticism, including a recent large study (Gray et al. 2018). Other studies, including a large study of 1050 individuals, found that the volumes of not only the left but also the right amygdala were positively associated with neuroticism (Holmes et al. 2012), and a unique older and relatively small study found a negative association between neuroticism and right amygdala volume (Omura et al. 2005). However, when systematic review and meta-analysis of voxel-based morphometry studies were performed, the most robust association was between a larger amygdala and high neuroticism, which is consistent with our results (Mincic 2015).

Other explanations for our highly significant association between amygdala volume and neuroticism may relate to the unique characteristics of our sample. Indeed, our study is the first to assess amygdala volume and neuroticism in a strictly female sample. Sex differences have been consistently found to substantially influence the association of amygdala volume with neuroticism, and previous studies have generally used sex as a confounding factor in the statistics. However, including only females in a studied sample may constitute a more powerful design to limit the effect of sex, and our results are therefore strongly consistent with the notion that left amygdala volume and neuroticism are specifically associated in females (Mincic 2015). Another reason could be that, although our sample is relatively small, the healthy controls displayed a large panel of neuroticism scores, ranging from 28 to 62, with individuals having various levels of neuroticism from low to high, whereas other studies that failed to find any association acknowledged that the levels of neuroticism observed were generally low to middle in their sample (Gray et al. 2018).

It may also appear surprising that on the one hand, amygdala volume positively correlates with neuroticism, a personality trait considered a risk factor for depression, while on the 
Fig. 1 Associations between neuroticism and left amygdala volume and brain pulsatility in healthy controls and in the depressed group
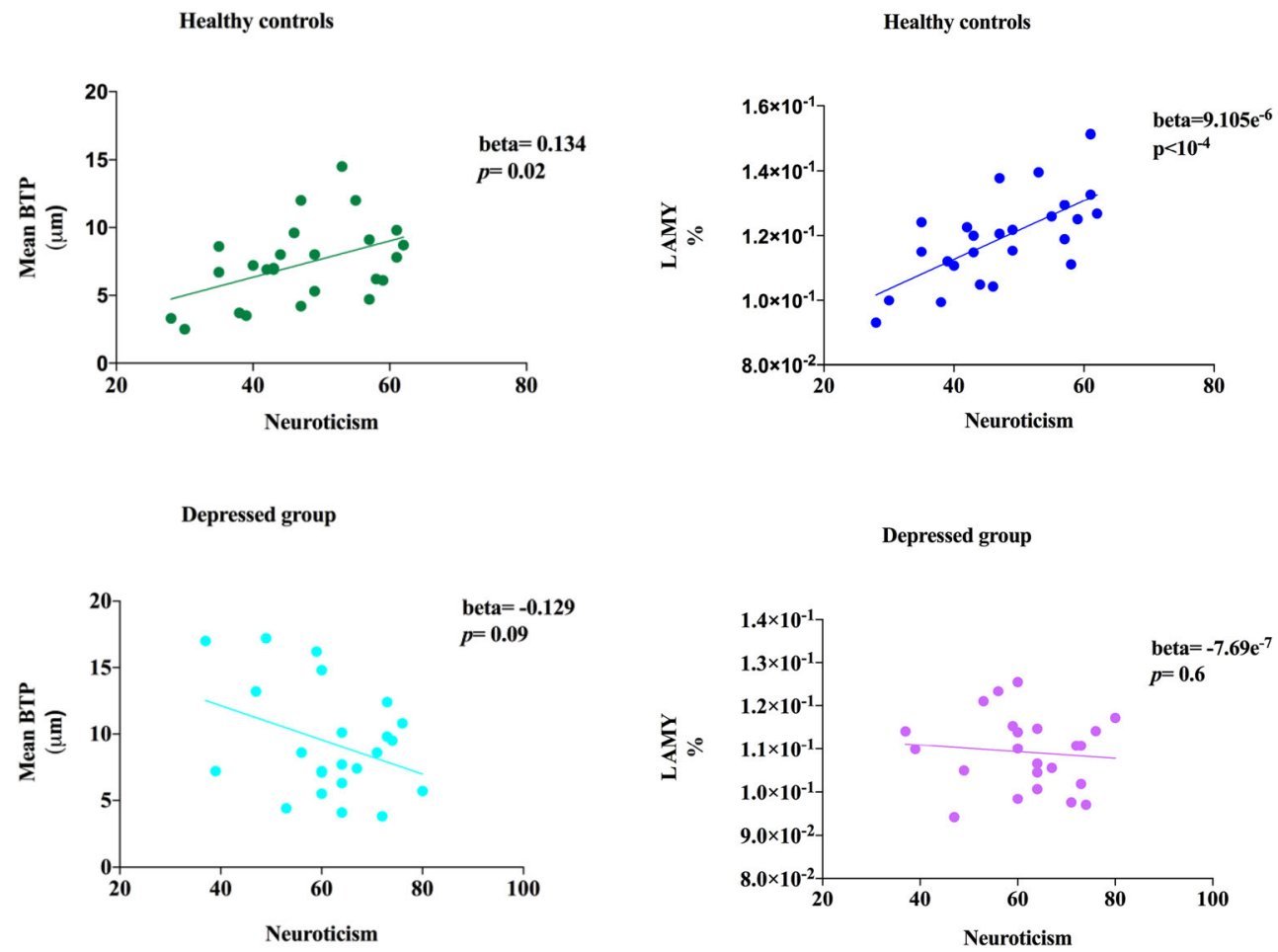

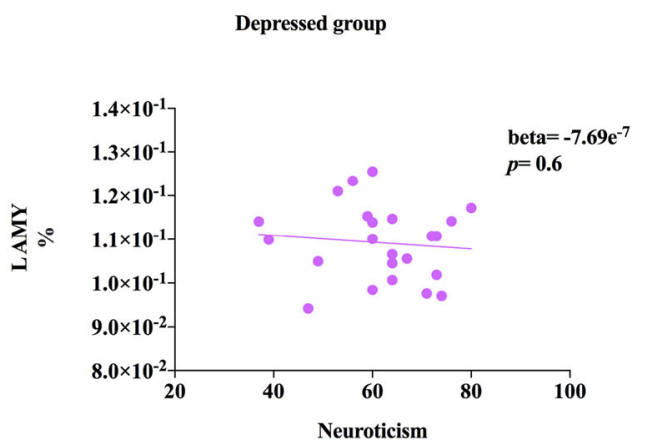

other hand, amygdala volume was lower in depressed individuals compared to controls, especially if we consider that our results exhibited a consistent trend of greater pulsatility with high neuroticism and in depressed individuals compared to controls. The associations of a smaller amygdala in depression have shown inconsistent results in the literature, although the general trend, as indicated notably in a meta-analysis (Hamilton et al. 2008), suggests a smaller amygdala with depression in studies that have included a certain proportion of unmedicated depressed individuals, which is consistent with our study (whereas a larger amygdala was found in studies with samples composed entirely of medicated depressed individuals, according to Hamilton et al. (2008)). In addition, other findings suggest that prolonged depression is more specifically associated with a smaller left amygdala, and a negative correlation has been identified between illness duration and amygdala volume (Zavorotnyy et al. 2018). Our study sample of depressed individuals had a relatively high illness duration (mean = 32 weeks; SD=36.5), which may account for the smaller left amygdala observed in our depressed individuals. In general, the amygdala has been considered a key structure involved in the pathophysiology of depression as a brain structure specifically involved in emotion regulation and fear conditioning (Kim et al. 2011). Finally, a larger amygdala may constitute a risk factor for depression (RomanczukSeiferth et al. 2014), which progressively reduces in volume, eventually reaching a smaller size as the disease progresses untreated while being restored in successful remission (Hamilton et al. 2008). However, this is still in the hypothesis stage, as no studies have investigated amygdala changes in individuals at risk of depression to a major depressive episode and eventually to remission.

Brain pulsatility may constitute different mechanisms in high neuroticism and in depression; it may not be directly involved in the pathophysiology of depression, but rather acts as an unspecific marker for a general hyperfunction of the brain in individuals who are prone to overreact to emotional stimuli, similar to the hyperactivity of the autonomic nervous system associated with neuroticism and depression. It is unlikely that the increase in brain pulsatility constitutes a causal mechanism for depression but rather may emerge as a consequence of depression-related metabolic changes or may alternatively constitute a compensatory mechanism to counteract the metabolic changes of depression and high neuroticism. Previous studies have suggested that cerebral blood flow $(\mathrm{CBF})$ is a major determinant for brain pulsatility and that greater brain pulsatility in high neuroticism may indicate an increase in $\mathrm{CBF}$ with high neuroticism, in accordance with previous studies that found greater $\mathrm{CBF}$ in high neuroticism using PET (Sutin et al. 2010). Moreover, greater brain pulsatility has been associated with increased cognitive activity (Angel et al. 2018; Kucewicz et al. 2007). Our results may therefore indicate that the greater brain pulsatility observed in higher neuroticism may be related to a trend of increased cognitive activity such as ruminations, which is a common feature of high neuroticism. Furthermore, some studies have suggested that excessive amplitudes in brain micromovements may constitute greater mechanical strains that can 
High Neuroticism - High amplitude BTP

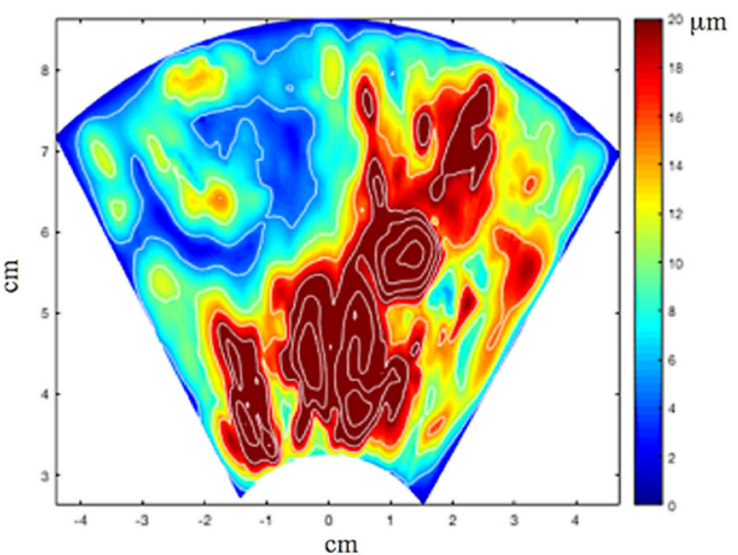

Low Neuroticism - Low amplitude BTP

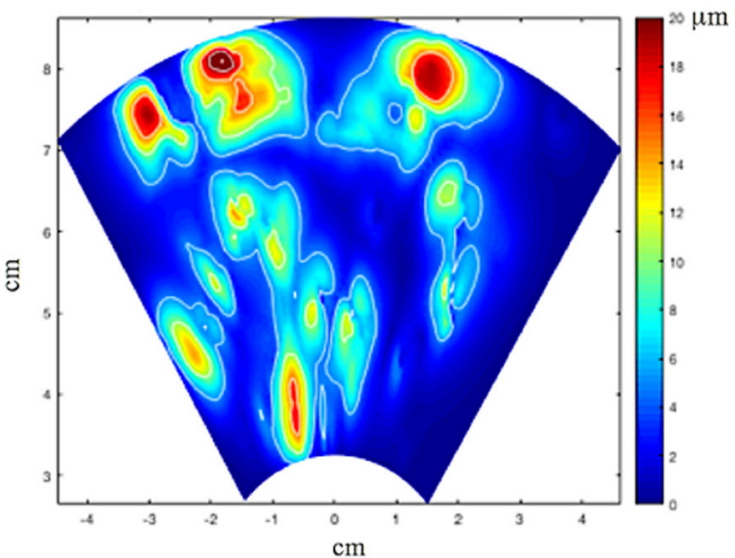

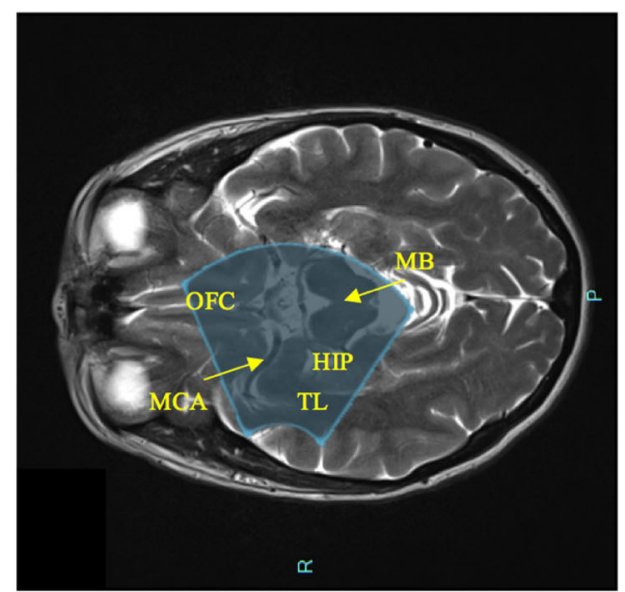

Fig. 2 Brain Tissue Pulsatility (BTP) color maps from two representative subjects in the nondepressed group. Consistent with our general results, subjects with high neuroticism (subject 1, left; neuroticism score 62) show high global BTP amplitudes, whereas subjects with low neuroticism (subject 2, right; neuroticism score 28) show low global BTP amplitudes. BTP color maps were obtained after applying the Horn-Schunck method to estimate the optical flow and displacement speed of each volume in the region of acquisition, the color maps of which show the maximum values. As shown on the bottom figure, the ultrasound beam of the TPI overlays parts of the temporal lobes (including parts of the hippocampus), the orbitofrontal cortex and the brainstem (midbrain). HIP: hippocampus; MB: midbrain; MCA: middle cerebral artery; OFC: orbitofrontal cortex; TL: temporal lobe

compensation of these disorders, whereas more specific neuronal changes may occur differentially in high neuroticism and in depression, as these two distinct disorders are possibly caused by different neuronal mechanisms.

Finally, it is possible that NeoPI may assess two different psychopathologies depending on whether individuals are experiencing major depression or not. Indeed, NeoPI has been specifically validated to assess personality traits, such as neuroticism, in the general community with no characterized psychopathology, such as major depression. In healthy controls, neuroticism has been consistently associated with a tendency for hyperreactivity to negative stimuli. In contrast, the underlying psychopathology of neuroticism as assessed with NeoPI is less clear in individuals experiencing a major depressive episode. Notably, while personality traits are supposed to be relatively stable in time, neuroticism was found to significantly change within the course of depression (Noordhof et al. the individual to cerebrovascular damage) both high neuroticism and depression as a consequence or a 
2018). Interestingly, recent findings suggest that the use of NeoPI to assess neuroticism in depressed individuals does not relate to the usual accepted notion of neuroticism as a tendency for hyperreactivity to negative stimuli, but rather that it could be more representative of the psychopathology of demoralization (Noordhof et al. 2018). Taken together, these results suggest that amygdala volume should be well correlated with neuroticism as a tendency for hyperreactivity to negative stimuli in nondepressed individuals, whereas it may not be correlated with demoralization as assessed with NeoPI in depressed individuals. Interestingly, we found a statistical trend for a negative correlation between brain pulsatility and neuroticism score in depressed individuals, suggesting that the psychopathology of demoralization may influence brain pulsatility in depression, with highly demoralized individuals showing lower pulsatility, possibly reflecting a slowing and a general despondency as clinical characteristics of a major depressive episode.

While meanBTP was significantly associated with neuroticism in healthy controls, we found that TCD-PI was not. This finding is consistent with our previous report of a greater meanBTP and no significant changes in TCD-PI in individuals with depression (Desmidt et al. 2017). MeanBTP corresponds to the averaged value of thousands of brain volume pulsations and indicates the mean global brain pulsatility, which is related to large and small artery pulsatility and the mechanical properties of the brain parenchyma. In contrast, TCD-PI indicates only the pulsatility of the MCA, the largest brain pulsatility. Therefore, our results suggest that neuroticism (similar to depression) is associated with changes in the general pulsatility of the brain, involving large and small arteries, as well as mechanical properties of the brain parenchyma but may not specifically involve changes in MCA pulsatility.

The limitations of our study notably concern the small sample size of our studied population and the cross-sectional design, which does not allow causal inference; additionally, it is unclear whether a larger amygdala and greater brain pulsatility precede or follow the development of neuroticism.

In conclusion, both larger amygdala volume and greater brain pulsatility appear to be involved in the brain physiology of neuroticism in healthy, nondepressed individuals. Because neuroticism is considered a risk factor for MDD, our results may suggest that a larger amygdala and greater brain pulsatility could constitute objective biomarkers involved in the risk of MDD occurrence. Our paper therefore provides potential objective biomarkers for the identification of subjects at risk for MDD, which could be included in longitudinal studies involving the general population while investigating baseline predictors of MDD incidence. Finally, while MRI is limited for daily clinical use, ultrasound technology shows rapid progress and may be particularly accurate and suitable for relatively easy clinical use. Specifically, TPI represents a low cost, easy-to-realize and noninvasive tool that may be suitable to investigate the physiology of individuals at risk for brain-related disorders such as depression and CVDs, and further studies may confirm the use of TPI in clinical practice.

Funding information This study received grants from the French National Research Agency (Agence Nationale de la Recherche: ANR2011- EMCO-005, 'EMPHILINE project') and the Planiol Foundation for Brain Study (Fondation Planiol pour le Cerveau), Varennes, France.

\section{Compliance with ethical standards}

\section{Conflict of interest The authors declare no conflict of interest.}

Ethical approval All procedures performed in studies involving human participants were in accordance with the ethical standards of the institutional and/or national research committee and with the 1964 Helsinki Declaration and its later amendments or comparable ethical standards.

Informed consent Informed consent was obtained from all participants included in the study.

\section{References}

Almas, A., Moller, J., Iqbal, R., \& Forsell, Y. (2017). Effect of neuroticism on risk of cardiovascular disease in depressed persons - a Swedish population-based cohort study. BMC Cardiovascular Disorders, 17(1), 185.

Angel, L., Bouazzaoui, B., Isingrini, M., Fay, S., Taconnat, L., Vanneste, S., Ledoux, M., Gissot, V., Hommet, C., Andersson, F., Barantin, L., Cottier, J. P., Pasco, J., Desmidt, T., Patat, F., Camus, V., \& Remenieras, J. P. (2018). Brain tissue pulsatility mediates cognitive and electrophysiological changes in normal aging: Evidence from ultrasound tissue pulsatility imaging (TPI). Brain and Cognition, 123, 74-80.

Clark, L. A., \& Watson D. (2008). Temperament: An organizing paradigm for trait psychology. In John OP, Robins RW, Pervin LA, editors. Handbook of personality: Theory and research (Guilford Press ; New York, pp. 265-286).

Costa, P. T., \& McCrae Jr., R. R. (1992). NEO PI-R professional manual.

Desmidt, T., Hachemi, M. E., Remenieras, J.-P., Lecomte, P., FerreiraMaldent, N., Patat, F., \& Camus, V. (2011). Ultrasound brain tissue Pulsatility is decreased in middle aged and elderly type 2 diabetic patients with depression. Psychiatry Research: Neuroimaging, 193(1), 63-64.

Desmidt, T., Brizard, B., Dujardin, P.-A., Ternifi, R., Réméniéras, J.-P., Patat, F., Andersson, F., Cottier, J. P., Vierron, E., Gissot, V., Kim, K., Aizenstein, H., el-Hage, W., \& Camus, V. (2017). Brain tissue Pulsatility is increased in midlife depression: A comparative study using ultrasound tissue Pulsatility imaging. Neuropsychopharmacology, 42, $2575-2582$.

Diagnostic and statistical manual of mental disorders : DSM-5. (2013).

Drevets, W., Videen, T., Price, J., Preskorn, S., Carmichael, S., \& Raichle, M. (1992). A functional anatomical study of unipolar depression. The Journal of Neuroscience, 12(9), 3628-3641.

Eysenck, H. J. (1967). The biological basis of personality.

Eysenck, H. J. (1990). Biological dimensions of personality.

Fischl, B., Salat, D., Busa, E., Albert, M., Dieterich, M., \& Haselgrove, C. (2002). Whole brain segmentation: Automated labeling of neuroanatomical structures in the human brain. Neuron, 33, 341-355. 
Fischl, B., Salat, D. H., van der Kouwe, A. J. W., Makris, N., Ségonne, F., Quinn, B. T., \& Dale, A. M. (2004a). Sequence-independent segmentation of magnetic resonance images. Mathematics in Brain Imaging, 23, S69-S84.

Fischl, B., van der Kouwe, A., Destrieux, C., Halgren, E., Ségonne, F., Salat, D. H., Busa, E., Seidman, L. J., Goldstein, J., Kennedy, D., Caviness, V., Makris, N., Rosen, B., \& Dale, A. M. (2004b). Automatically parcellating the human cerebral cortex. Cerebral Cortex, 14(1), 11-22.

Gray, J. C., Owens, M. M., Hyatt, C. S., \& Miller, J. D. (2018). No evidence for morphometric associations of the amygdala and hippocampus with the five-factor model personality traits in relatively healthy young adults. PLoS One, 13(9), e0204011-e0204011.

Hamilton, J., Siemer, M., \& Gotlib, I. (2008). Amygdala volume in major depressive disorder: A meta-analysis of magnetic resonance imaging studies. Molecular Psychiatry, 13, 993-1000.

Ho, T. C., Wu, J., Shin, D. D., Liu, T. T., Tapert, S. F., Yang, G., Connolly, C. G., Frank, G. K. W., Max, J. E., Wolkowitz, O., Eisendrath, S., Hoeft, F., Banerjee, D., Hood, K., Hendren, R. L., Paulus, M. P., Simmons, A. N., \& Yang, T. T. (2013). Altered cerebral perfusion in executive, affective, and motor networks during adolescent depression. Journal of the American Academy of Child and Adolescent Psychiatry, 52(10), 1076-1091.e2. https:// doi.org/10.1016/j.jaac.2013.07.008.

Holmes, A. J., Lee, P. H., Hollinshead, M. O., Bakst, L., Roffman, J. L., Smoller, J. W., \& Buckner, R. L. (2012). Individual differences in amygdala-medial prefrontal anatomy link negative affect, impaired social functioning, and polygenic depression risk. The Journal of Neuroscience, 32(50), 18087-18100.

Kempton, M. J., Salvador, Z., Munafò, M. R., Geddes, J. R., Simmons, A., Frangou, S., \& Williams, S. C. (2011). Structural neuroimaging studies in major depressive disorder. Meta-analysis and comparison with bipolar disorder, 675-690.

Kim, M. J., Loucks, R. A., Palmer, A. L., Brown, A. C., Solomon, K. M., Marchante, A. N., \& Whalen, P. J. (2011). The structural and functional connectivity of the amygdala: From normal emotion to pathological anxiety. Behavioural Brain Research, 223(2), 403-410.

Kotov, R., Gamez, W., Schmidt, F., \& Watson, D. (2010). Linking "big" personality traits to anxiety, depressive, and substance use disorders: A meta-analysis. Psychological Bulletin, 136(5), 768-821.

Kucewicz, J. C., Huang, L., \& Beach, K. W. (2004). Plethysmographic arterial waveform strain discrimination by Fisher's method. Ultrasound in Medicine and Biology, 30(6), 773-782.

Kucewicz, J. C., Dunmire, B., Leotta, D. F., Panagiotides, H., Paun, M., \& Beach, K. W. (2007). Functional tissue Pulsatility imaging of the brain during visual stimulation. Ultrasound in Medicine and Biology, 33(5), 681-690.

Kucewicz, J. C., Dunmire, B., Giardino, N. D., Leotta, D. F., Paun, M., Dager, S. R., \& Beach, K. W. (2008). Tissue Pulsatility imaging of cerebral Vasoreactivity during hyperventilation. Ultrasound in Medicine and Biology, 34(8), 1200-1208.

Liao, W., Wang, Z., Zhang, X., Shu, H., Wang, Z., Liu, D., \& Zhang, Z. (2017). Cerebral blood flow changes in remitted early- and lateonset depression patients. Oncotarget, 8(44), 76214-76222. https://doi.org/10.18632/oncotarget.19185.

Lui, S., Parkes, L. M., Huang, X., Zou, K., Chan, R. C. K., Yang, H., Zou, L., Li, D., Tang, H., Zhang, T., Li, X., Wei, Y., Chen, L., Sun, X., Kemp, G. J., \& Gong, Q. Y. (2009). Depressive disorders: Focally altered cerebral perfusion measured with arterial spin-labeling MR imaging. Radiology, 251(2), 476-484.
Mincic, A. M. (2015). Neuroanatomical correlates of negative emotionality-related traits: A systematic review and meta-analysis. Neuropsychologia, 77, 97-118.

Noordhof, A., Kamphuis, J. H., Sellbom, M., Eigenhuis, A., \& Bagby, R. M. (2018). Change in self-reported personality during major depressive disorder treatment: A reanalysis of treatment studies from a demoralization perspective. Personality Disorders, 9(1), 93-100.

Omura, K., Todd Constable, R., \& Canli, T. (2005). Amygdala gray matter concentration is associated with extraversion and neuroticism. Neuroreport, 16(17), 1905-1908.

Ormel, J., Jeronimus, B. F., Kotov, R., Riese, H., Bos, E. H., Hankin, B., Rosmalen, J. G. M., \& Oldehinkel, A. J. (2013). Neuroticism and common mental disorders: Meaning and utility of a complex relationship. Clinical Psychology Review, 33(5), 686-697.

Reuter, M., Rosas, H. D., \& Fischl, B. (2010). Highly accurate inverse consistent registration: A robust approach. NeuroImage, 53(4), 1181-1196.

Romanczuk-Seiferth, N., Pöhland, L., Mohnke, S., Garbusow, M., Erk, S., Haddad, L., Grimm, O., Tost, H., Meyer-Lindenberg, A., Walter, H., Wüstenberg, T., \& Heinz, A. (2014). Larger amygdala volume in first-degree relatives of patients with major depression. NeuroImage. Clinical, 5, 62-68.

Schmaal, L., Veltman, D. J., van Erp, T. G. M., Sämann, P. G., Frodl, T., Jahanshad, N., et al. (2015). Subcortical brain alterations in major depressive disorder: Findings from the ENIGMA major depressive disorder working group. Molecular Psychiatry, 21, 806.

Ségonne, F., Dale, A. M., Busa, E., Glessner, M., Salat, D., Hahn, H. K., \& Fischl, B. (2004). A hybrid approach to the skull stripping problem in MRI. NeuroImage, 22(3), 1060-1075.

Sutin, A. R., Beason-Held, L. L., Dotson, V. M., Resnick, S. M., \& Costa Jr., P. T. (2010). The neural correlates of neuroticism differ by sex prospectively mediate depressive symptoms among older women. Journal of Affective Disorders, 127(1), 241-247.

Ternifi, R., Cazals, X., Desmidt, T., Andersson, F., Camus, V., Cottier, J.P., Patat, F., \& Remenieras, J. P. (2014). Ultrasound measurements of brain tissue Pulsatility correlate with the volume of MRI whitematter Hyperintensity. Journal of Cerebral Blood Flow \& Metabolism, 34(6), 942-944.

Tsao, C. W., Seshadri, S., Beiser, A. S., Westwood, A. J., DeCarli, C., Au, R., Himali, J. J., Hamburg, N. M., Vita, J. A., Levy, D., Larson, M. G., Benjamin, E. J., Wolf, P. A., Vasan, R. S., \& Mitchell, G. F. (2013). Relations of arterial stiffness and endothelial function to brain aging in the community. Neurology, 81(11), 984-991.

Wagshul, M. E., Eide, P. K., \& Madsen, J. R. (2011). The pulsating brain: A review of experimental and clinical studies of intracranial pulsatility. Fluids and Barriers of the CNS, 8(1), 5.

Wei, L., Duan, X., Zheng, C., Wang, S., Gao, Q., Zhang, Z., Lu, G., \& Chen, H. (2014). Specific frequency bands of amplitude lowfrequency oscillation encodes personality. Human Brain Mapping, 35(1), 331-339.

Yamasue, H., Abe, O., Suga, M., Yamada, H., Inoue, H., et al. (2008). Gender- common and-specific neuroanatomical basis of human anxiety-related personality traits. Cereb Cortex, 18(1), 46-52.

Zavorotnyy, M., Zöllner, R., Schulte-Güstenberg, L. R., Wulff, L., Schöning, S., Dannlowski, U., Kugel, H., Arolt, V., \& Konrad, C. (2018). Low left amygdala volume is associated with a longer duration of unipolar depression. Journal of Neural Transmission, 125(2), 229-238.

Publisher's note Springer Nature remains neutral with regard to jurisdictional claims in published maps and institutional affiliations. 


\section{Terms and Conditions}

Springer Nature journal content, brought to you courtesy of Springer Nature Customer Service Center GmbH ("Springer Nature").

Springer Nature supports a reasonable amount of sharing of research papers by authors, subscribers and authorised users ("Users"), for smallscale personal, non-commercial use provided that all copyright, trade and service marks and other proprietary notices are maintained. By accessing, sharing, receiving or otherwise using the Springer Nature journal content you agree to these terms of use ("Terms"). For these purposes, Springer Nature considers academic use (by researchers and students) to be non-commercial.

These Terms are supplementary and will apply in addition to any applicable website terms and conditions, a relevant site licence or a personal subscription. These Terms will prevail over any conflict or ambiguity with regards to the relevant terms, a site licence or a personal subscription (to the extent of the conflict or ambiguity only). For Creative Commons-licensed articles, the terms of the Creative Commons license used will apply.

We collect and use personal data to provide access to the Springer Nature journal content. We may also use these personal data internally within ResearchGate and Springer Nature and as agreed share it, in an anonymised way, for purposes of tracking, analysis and reporting. We will not otherwise disclose your personal data outside the ResearchGate or the Springer Nature group of companies unless we have your permission as detailed in the Privacy Policy.

While Users may use the Springer Nature journal content for small scale, personal non-commercial use, it is important to note that Users may not:

1. use such content for the purpose of providing other users with access on a regular or large scale basis or as a means to circumvent access control;

2. use such content where to do so would be considered a criminal or statutory offence in any jurisdiction, or gives rise to civil liability, or is otherwise unlawful;

3. falsely or misleadingly imply or suggest endorsement, approval, sponsorship, or association unless explicitly agreed to by Springer Nature in writing;

4. use bots or other automated methods to access the content or redirect messages

5. override any security feature or exclusionary protocol; or

6. share the content in order to create substitute for Springer Nature products or services or a systematic database of Springer Nature journal content.

In line with the restriction against commercial use, Springer Nature does not permit the creation of a product or service that creates revenue, royalties, rent or income from our content or its inclusion as part of a paid for service or for other commercial gain. Springer Nature journal content cannot be used for inter-library loans and librarians may not upload Springer Nature journal content on a large scale into their, or any other, institutional repository.

These terms of use are reviewed regularly and may be amended at any time. Springer Nature is not obligated to publish any information or content on this website and may remove it or features or functionality at our sole discretion, at any time with or without notice. Springer Nature may revoke this licence to you at any time and remove access to any copies of the Springer Nature journal content which have been saved.

To the fullest extent permitted by law, Springer Nature makes no warranties, representations or guarantees to Users, either express or implied with respect to the Springer nature journal content and all parties disclaim and waive any implied warranties or warranties imposed by law, including merchantability or fitness for any particular purpose.

Please note that these rights do not automatically extend to content, data or other material published by Springer Nature that may be licensed from third parties.

If you would like to use or distribute our Springer Nature journal content to a wider audience or on a regular basis or in any other manner not expressly permitted by these Terms, please contact Springer Nature at

onlineservice@springernature.com 